\title{
Motivational Interviewing with Parents of Overweight Children: Study Design and Methods for the NOURISH+MI Study
}

\author{
Melanie K. Bean, PhD ${ }^{a}$, Amy J. Jeffers, $\mathbf{M S}^{b}$, Carrie B. Tully, $\mathbf{M S}^{b}$, Laura M. Thornton, $\mathbf{P h D}^{\mathrm{c}}$, \\ and Suzanne E. Mazzeo, PhD ${ }^{\mathrm{b}, \mathrm{a}}$ \\ aDepartment of Pediatrics, Children's Hospital of Richmond at Virginia Commonwealth University

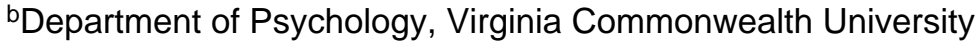 \\ 'Department of Psychiatry, University of North Carolina, Chapel Hill
}

\begin{abstract}
There is an urgent need for innovative approaches to pediatric obesity treatment. There is also a demand for targeted strategies that reduce attrition and improve treatment adherence. Intervening exclusively with parents of overweight children is a novel approach with demonstrated efficacy in reducing child body mass index (BMI) percentile. Motivational interviewing (MI), a brief communication style for exploring and resolving ambivalence about behavior change, might enhance treatment engagement when implemented as part of obesity interventions. The aim of this report is to provide the rationale and methods for a novel study of MI with parents in the treatment of their children's overweight. We designed and are currently implementing NOURISH+MI, a randomized controlled trial examining the feasibility and efficacy of an adjunct values-based MI intervention, implemented within a culturally-tailored parent intervention for overweight children ages 5-11 years, $\mathrm{NOURISH}^{+}$(Nourishing Our Understanding of Role modeling to Improve Support and Health). Specifically, we are randomly assigning 60 parents to this adjunctive treatment, and investigating if adding two MI sessions prior to the $\mathrm{NOURISH}^{+}$group intervention will enhance treatment effects. We will be able to compare NOURISH+MI participants with those from the two NOURISH ${ }^{+}$treatment conditions (NOURISH ${ }^{+}$and control). We hypothesize that children whose parents participate in NOURISH+MI will demonstrate lower attrition and greater adherence with $\mathrm{NOURISH}^{+}$, ultimately leading to greater treatment effects, compared with children whose parents are randomized to $\mathrm{NOURISH}^{+}$or a control group. Findings will contribute to the emerging literature examining the efficacy of MI within pediatric obesity interventions.
\end{abstract}

\section{Keywords}

motivational interviewing; pediatric obesity; treatment adherence; attrition

\footnotetext{
(C) 2014 Elsevier Inc. All rights reserved.

Corresponding Author: Melanie K. Bean, Assistant Professor, Department of Pediatrics, Children's Hospital of Richmond at VCU, 2305 N. Parham Road, Suite 1, Richmond, VA 23229, Tel: 804-527-4765, mkbean@vcu.edu.

Publisher's Disclaimer: This is a PDF file of an unedited manuscript that has been accepted for publication. As a service to our customers we are providing this early version of the manuscript. The manuscript will undergo copyediting, typesetting, and review of the resulting proof before it is published in its final citable form. Please note that during the production process errors may be discovered which could affect the content, and all legal disclaimers that apply to the journal pertain.
} 


\section{Introduction}

Lifestyle interventions are recommended to reduce the significant burden of pediatric obesity. ${ }^{1-7}$ Low treatment adherence and high attrition, however, are frequently encountered, particularly when targeting underserved populations, reducing the effectiveness of these interventions. ${ }^{8-10}$ Innovative strategies to enhance retention and treatment adherence are needed. ${ }^{10,11}$

\section{Parent Involvement in Obesity Treatment}

Parent involvement in the treatment of overweight youth results in improved weight outcomes, particularly when parental behavior change is expected. ${ }^{12}$ Research examining parent-exclusive interventions found equal or greater reductions in childhood overweight compared with family-based interventions; they were also more cost-effective. ${ }^{13-15}$ However, these studies targeted predominately White, intact families and might not generalize to African Americans. Responding to the need for intervention models targeting the unique cultural and pragmatic needs of African American families, ${ }^{16}$ we previously developed and piloted NOURISH (Nourishing $\underline{\text { Our }}$ Understanding of Role-modeling to

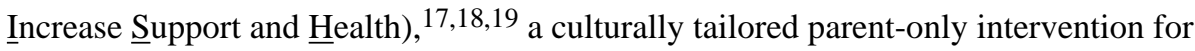
overweight children ages 6-11 years. We made modifications based on this pilot and are currently implementing a larger trial, NOURISH+, with parents of 5-11 year old overweight children, providing the foundation for the current study.

\section{Increases Treatment Engagement}

Many strategies (e.g., use of incentives, implementing culturally sensitive interventions, and making repeated participant contact) are recommended to address the notorious problems of high attrition and low treatment adherence. ${ }^{9}$ It is likely that ambivalence and low internal motivation for behavioral change contribute to these challenges. ${ }^{20}$ Thus, targeted strategies addressing internal barriers to participation are needed. One innovative strategy demonstrated to increase treatment engagement in other behavioral domains is Motivational Interviewing (MI). ${ }^{21}$

MI is a communication technique that reduces ambivalence about behavior change in a patient-centered, empathic manner. ${ }^{20}$ By facilitating exploration of advantages to change and eliciting patient-determined reasons for change, internal motivation and readiness to make behavioral modifications are increased. ${ }^{20}$ Studies have demonstrated the utility of MI across behavioral domains. ${ }^{21-24}$ Although very few randomized trials specifically target pediatric obesity, research suggests the potential of this approach to elicit positive outcomes. ${ }^{23,25-28}$ For example, in a non-randomized trial of MI delivered in primary care to parents of overweight children, trends (nonsignificant) towards decreased BMI percentile were found in children whose parents participated in MI versus standard of care. ${ }^{25}$ In the $M I$ Values study, we implemented a randomized controlled trial (RCT) of MI within an adolescent obesity treatment. ${ }^{29}$ Main findings were that, although both groups reduced BMI z-scores, MI participants had greater treatment adherence and remained in treatment eight weeks longer than controls,${ }^{30}$ suggesting the benefits of MI in addressing adherence and retention among obese adolescents. Implementing MI with parents for the treatment of their 
children's overweight has not been well-examined and might be an effective strategy to enhance treatment effects in this population. Only two studies have conducted MI with parents of overweight children (both in office-based settings). ${ }^{25,28}$ The current study addresses this need, and implements an MI intervention in a community setting as a "pretreatment" for parents of overweight children. We report the design and methods of NOURISH+MI, a pilot adjunctive MI intervention for parents of overweight children.

\section{Specific Aims and Hypotheses}

The NOURISH+MI study has four aims and corresponding hypotheses:

1. Pilot the feasibility and efficacy of a brief MI intervention as an adjunct to the RCT of $\mathrm{NOURISH}^{+}$, a parent intervention for pediatric overweight. Examine the impact of NOURISH+MI on attrition and adherence.

Our primary hypothesis is that parents who participate in NOURISH+MI will demonstrate lower attrition and greater treatment adherence, as measured by attendance at group sessions, than parents in $\mathrm{NOURISH}^{+}$without MI.

2. Examine the efficacy of NOURISH+MI on child BMI percentile changes (the primary NOURISH ${ }^{+}$outcome), and secondary outcomes of child dietary intake and physical activity.

We hypothesize that children whose parents participate in NOURISH+MI will achieve greater decreases in BMI percentile compared with children whose parents are enrolled in $\mathrm{NOURISH}^{+}$or a control group. Further, children whose parents participate in NOURISH+MI will achieve greater improvements in dietary intake and physical activity than children whose parents are enrolled in $\mathrm{NOURISH}^{+}$or a control group.

3. Evaluate the impact of NOURISH+MI on adult participants, including parental BMI change and improvements in dietary intake and physical activity levels.

We hypothesize that parents who participate in NOURISH+MI will achieve greater BMI decreases and greater improvements in dietary behavior and physical activity than children whose parents are enrolled in $\mathrm{NOURISH}^{+}$or a control group.

4. Identify putative mediators and moderators of treatment outcome.

We will examine the influence of putative moderators (including ethnicity and gender of children) and mediators (e.g., parent BMI, parental readiness to change) on outcomes. These hypothesis-generating analyses will to inform future translational research based upon this study's findings.

\section{Study Design and Methods}

\section{Participants}

To qualify for $\mathrm{NOURISH}^{+}$(and thus the NOURISH+MI study), parents/caregivers must be at least 18 years old and have a child between the ages of 5 and 11 years with a BMI $\geq$ the $85^{\text {th }}$ percentile who primarily resides in the caregiver's home. This age range was extended 
to age 5 years (was previously ages 6 to 11 years in the NOURISH pilot) due to the expressed need for earlier intervention to prevent progression or development of obesity. Participants also need to speak English, be able to follow basic instructions, and perform simple exercises. Caregivers are ineligible if they are non-ambulatory, pregnant, have a medical condition that might be negatively impacted by exercise, or have a psychiatric diagnosis that would impair their ability to respond to assessments or participate in a group. Parents whose children have an underlying medical etiology of obesity (e.g., Prader-Willi Syndrome) are also ineligible.

\section{Consent and Assent Procedures}

$\mathrm{NOURISH}^{+}$and NOURISH+MI study procedures are approved by the Institutional Review board of Virginia Commonwealth University. Participants are asked to provide their verbal consent prior to beginning the screening interview. Similarly, prior to beginning the baseline assessment, adult participants provide written consent for both their participation, and their child's (via the parental consent form). Children provide written assent.

\section{Randomization Methods and Experimental Design}

Figure 1 presents an overview of NOURISH+MI embedded within the larger trial. NOURISH+MI uses a repeated measures design, with assessments at pre-test, post-test, and 4-month follow up. Recruitment occurs in waves. Screened, eligible participants are randomized (using a random number generator) in blocks of 60, to participate in either NOURISH+MI or NOURISH ${ }^{+}$, using a 1 (NOURISH+MI) to $2\left(\mathrm{NOURISH}^{+}\right)$ratio. Those randomized to the $\mathrm{NOURISH}^{+}$trial are subsequently randomly assigned to either $\mathrm{NOURISH}^{+}$or control. Thus, a $3\left(\mathrm{NOURISH}+\mathrm{MI}, \mathrm{NOURISH}^{+}\right.$, control) $\times 3$ (pre-test, posttest, 4-month follow-up) repeated measures design will be analyzed.

\section{Overview of NOURISH ${ }^{+}$and Rationale Behind NOURISH+MI}

$\mathrm{NOURISH}^{+}$methods have been described in detail elsewhere. ${ }^{18} \mathrm{Briefly} \mathrm{NOURISH}^{+}$is a 6 session group-based intervention, grounded in Social Cognitive Theory, ${ }^{31}$ that targets parents exclusively as the agent of change for their child(ren) with overweight or obesity. $\mathrm{NOURISH}^{+}$emphasizes parental role modeling of healthy dietary and physical activity behaviors and fosters parent self-efficacy for health behavior change. NOURISH $^{+}$uses guided goal-setting and self-monitoring to set parent goals for change, with the intent to improve the whole family's health, due to the strong influence of parent behaviors on children's behaviors. ${ }^{32}$ The program is culturally sensitive for African American families (although families of all racial/ethnic backgrounds are eligible). This cultural sensitivity includes modifying traditional meals with healthy adaptations; acknowledging and incorporating ethnic differences in body image ideals; acknowledging the special financial and pragmatic challenges facing single parent families; and recognizing and valuing extended kinship networks. ${ }^{33}$

In the NOURISH pilot, parents were randomly assigned to either NOURISH or a control group. Compared with controls, intervention participants significantly reduced child BMI percentile $(\mathrm{p}=0.02) .{ }^{34}$ However, strategies to reduce attrition $(32 \%$ at post-test, $66 \%$ at 6 month follow-up) and increase treatment adherence (e.g., $48 \%$ of NOURISH participants 
attended $<50 \%$ of sessions) were needed. Procedures were modified in the ongoing larger trial of NOURISH ${ }^{+}$to respond to these issues (i.e., more hands-on experiences and changing locations). We designed NOURISH+MI as a brief, adjunctive MI intervention to enhance treatment effects further through reduced attrition and improved adherence.

\section{Adjunctive MI Intervention}

Parents of overweight children (who are typically overweight themselves ${ }^{4}$ ) likely manifest ambivalence about making behavioral changes. Thus, the addition of a brief MI intervention might enhance treatment engagement and improve outcomes. After participating in MI, parents might be more ready to make behavioral changes and adhere to treatment to benefit themselves and their overweight child(ren). We will be able to examine if participation in NOURISH+MI enhances outcomes in NOURISH ${ }^{+}$.

NOURISH+MI methods are identical to the NOURISH ${ }^{+}$methods described above, with the addition of two brief ( 20 minute) MI sessions. All MI sessions are conducted by trained MI interventionists, independent from NOURISH ${ }^{+}$to reduce the risk of contamination. Sessions are audio recorded and coded for MI adherence, using a standardized measure (described below). ${ }^{35}$ Parents first learn that NOURISH ${ }^{+}$is a parent-exclusive study at the telephone screening. In the NOURISH pilot, $~ 50 \%$ of families who successfully completed the telephone screen did not attend the orientation session (43\% of whom agreed to attend; $8 \%$ declined participation), highlighting a significant loss of potential participants, and a common point of attrition across obesity treatments. ${ }^{10}$ Using MI at this stage to explore ambivalence and readiness to participate might be an effective strategy to engage parents and increase readiness to enter treatment, thereby reducing attrition. Thus, in a two-week window after the telephone screening, MI interventionists conduct Session 1 (via telephone). This call is the participants' first interaction with the MI interventionist, although they will have previously been in contact via telephone with a study team member for the telephone screen / verbal consent. The first MI contact is made via telephone to match the modality of this initial study contact, with minimal patient burden, with the goal to increase in-person attendance at the baseline assessment (and subsequent intervention sessions). Parents then attend the orientation and complete baseline assessments. MI Session 2 is scheduled at this time, and is conducted in-person during the two-week window prior to beginning the NOURISH $^{+}$group treatment. All MI participants complete the NOURISH ${ }^{+}$treatment, as described above.

Research indicates that manualized MI is less efficacious than MI without a manual because interventionists' adherence to the manual, at times, sacrifices competent MI practice. ${ }^{21}$ For NOURISH+MI, a "session roadmap" is used to guide the intervention and includes a flexible outline of the goals for the encounter. Session 1 focuses on exploring participants' ambivalence about entering treatment targeting their child's weight, eliciting and exploring pros and cons of change, parent-determined reasons for change (or not changing), and barriers and facilitators to change. Interventionists review/discuss participants' motivation to make behavioral changes, using strategies such as importance and confidence rulers, decisional balance, and complex reflections; (see ${ }^{36,37}$ ). The same interventionist conducts both MI sessions. Session 2 builds on the relationship established and content explored in 
Session 1. Upon arriving for this visit, parents complete a values checklist (see Figure 2) selecting values that are important to them; of those checked, parents then select their top three values. In the MI session which immediately follows completion of this checklist, the interventionist facilitates developing discrepancy between parent-selected top three values and current family/child health behaviors, with an exploration of how participation in a parent-based treatment for their child's weight might resolve the identified discrepancy. ${ }^{38}$ This exercise was selected based on our prior work in MI Values, ${ }^{29}$ which found that adolescents with obesity who participated in a values-based MI treatment were more adherent to a multidisciplinary lifestyle intervention than adolescents enrolled in a control treatment. ${ }^{30}$ Throughout both MI sessions, the interventionists are MI-adherent, using open questions, reflections and affirmations to express empathy, develop discrepancy, highlight parent autonomy, and support parents' self-efficacy for change in a non-confrontational, directive manner. At the conclusion of each session, interventionists summarize what parents said is important to them, empathize with difficulties noted, highlight parentreported reasons for change, and reflect readiness and willingness to change, with the overall goal to enhance participation in NOURISH ${ }^{+} .22$ Through use of MI, parent ambivalence is thus explored and resolved prior to participation, to increase engagement in $\mathrm{NOURISH}^{+}$.

\section{Measures}

All measures described below are completed at baseline, post-testing, and 4-month followup (see Table 1). The parent/caregiver enrolled in the study completes all measures each time his/her child does so. Measures are identical to those implemented in NOURISH ${ }^{+}$, except where noted:

\section{Parent Measures}

Parental Readiness to Change-An 11-item survey assesses parent readiness to make lifestyle changes to help their child lose weight. ${ }^{39}$ This survey is conducted via telephone at the initial screening and in-person at post-testing and 4-month follow-up. Algorithms based on Kristal et al. ${ }^{40}$ and described in Rhee et al. ${ }^{39}$ are used to determine parental stage of change (i.e., Precontemplation through Maintenance).

Child Feeding Questionnaire (CFQ)—Parental approaches to and attitudes about feeding their children are measured by the CFQ, which has yielded reliable and valid scores in parents with elementary age children. ${ }^{41}$ This measure includes seven subscales: perceived responsibility, perceived parent weight, perceived child weight, concern about child weight, pressure to eat, monitoring, and restriction.

Anthropometric measures-Height is measured to the nearest $1 / 4 \mathrm{~cm}$ using a stadiometer. Weight is measured to the nearest $1 / 4 \mathrm{~kg}$ using a digital bariatric scale. These data are used to calculate BMI. Hip (maximum girth, above the gluteal fold), abdominal (level of the umbilicus), and waist (narrowest part of the torso above the umbilicus and below the xiphoid process) circumferences are assessed using an anthropometric measuring tape using standardized procedures. Data are used to calculate waist-hip ratio. Percent body fat is determined by bioelectrical impedance. 
7-day Physical Activity Recall (PAR)—Parental physical activity is assessed using the 7-day PAR. This interview measure asks respondents the amount of time they spent in sleep and moderate and vigorous activities during the previous 7 days. ${ }^{42}$ Previous research produced same-day reliabilities across interviewers of $0.86 .{ }^{43}$

24-Hour Food Record-Parents are trained during their scheduling interview to complete a dietary record for one weekday. They receive a food record form to track foods and beverages consumed; portions; time of day consumed; and details on food preparation for the weekday prior to their assessment. At their assessment appointment, a trained research assistant (e.g., dietetic intern) interviews parents to review dietary intake, how foods were prepared, and obtain a portion size estimate using food models. Foods are analyzed using the Nutrition Data System Software (NDS-R), ${ }^{44,45}$ which provides data organized by food group servings, calories, and nutrient content.

Family Eating and Exercise Behaviors-Items used in previous work by NeumarkSztainer et al. ${ }^{46}$ are used to assess families' eating and weight-related habits. Specifically, the frequency of family meals, fast food consumption, and watching television during meals are assessed. Parents are also asked about fruit and vegetable and sugar sweetened beverage availability and consumption in the home. Finally, parents complete items assessing their encouragement of healthy food consumption, physical activity, and dieting.

\section{Child Measures}

Anthropometric Measures-Height, weight, waist-hip ratio and percent body fat are measured using standardized methodology described above (Parent Measures). Height and weight data are used to calculate children's BMI, which are plotted on the CDC Growth Charts ${ }^{47}$ to obtain BMI percentile for age and gender.

24-Hour Food Record-Parents are trained to complete a dietary record for their children on one weekday, using the methodology described above (Parent Measures). For young children, parent report of intake is more valid than self-report and demonstrates convergence with observational methods of diet. ${ }^{48} \mathrm{~A}$ trained research assistant or dietetic intern interviews children and parents to review the record and clarify portions and preparation methods.

A trained Research Assistant verbally administers the following assessments to children (with parental assistance):

Pediatric Health-Related Quality of Life (PedsQL 4.0)—The PedsQL ${ }^{49,50}$ assesses perceptions of how health affects daily life in four areas: physical, emotional, social, and school. Reliability (alpha) is high, and the PedsQL discriminates effectively between healthy children and those with health problems. ${ }^{49,50}$

7-day Physical Activity Recall (PAR)—This interview is completed with children (with parental-assistance) as described above (Parent Measures). ${ }^{42,43}$ Previous research produced same-day reliabilities across interviewers of $0.86 .{ }^{43}$ 
Pubertal Status-Children (8 or older) complete a self-assessment of pubertal status using a pictorial measure. ${ }^{51}$ This measure was significantly associated with results of physical examinations and is recommended for use in studies where clinical examinations are not feasible. ${ }^{51}$ For children $<8$ years, we ask parents to report children's pubertal status.

Child Sugar Sweet Beverage and Fast Food Intake-Parents assist children in reporting weekly sugar-sweetened beverages consumption and fast food consumption.

\section{Additional Measures}

Motivational Interviewing Treatment Integrity Code 3.1 (MITI 3.1)-The MITI 3.1 is a behavioral coding system that is used to monitor treatment fidelity of MI sessions. ${ }^{35}$ Trained raters code randomly selected 20 minute segments of each MI session. Inter-rater reliabilities (using intraclass correlations [ICCs]) were assessed at study onset and continue to be assessed throughout the study (see ${ }^{36}$ ). The MITI 3.1 was also used to determine clinician MI competence during interventionist training and monitor fidelity throughout the study duration. Specifically, interventionists had to reach or exceed competency thresholds described in the MITI 3.1 to begin study participation.

Working Alliance Inventory (WAI)-MI interventionists and parents also complete the WAI, a reliable and valid measure of the working relationship. ${ }^{52}$ The MITI 3.1 and WAI are used with the MI participants only. Attrition, attendance, and fidelity to NOURISH ${ }^{+}$are also monitored.

\section{Intervention fidelity}

Interventionist training and ongoing monitoring are important to ensure intervention fidelity. Interventionists attended a two-day (12 hour) training in MI to learn the spirit and principles of MI practice. To achieve initial proficiency, each interventionist conducted 30 hours of practice with weekly supervision using feedback of audiotaped sessions until they met or exceeded competency thresholds set in the MITI 3.1 and as judged by an MI expert. During the trial, a licensed clinical psychologist with expertise in MI (M.K.B.) provided weekly supervision to interventionists. These meetings included group observation of a recorded MI session with live feedback, as well as skill rehearsal to ensure continued competence and adherence to the clinical intervention. Table 2 presents initial interventionist ratings prior to study initiation.

Raters were rigorously trained to code MI sessions using the MITI 3.1. Raters attended a multi-day training which included an overview of intervention fidelity, instruction on how to parse and code clinician utterances and how to use the MITI 3.1. Raters participated in more than 30 hours of group rating sessions, which included rating pre-scored gold-standard transcripts from motivationalinterviewing.org and comparing team ratings to those provided on this training website. Feedback was provided immediately to raters and questions addressed. Audiotaped sessions were independently coded by three raters until satisfactory intraclass correlations (ICCs) were obtained (e.g., 0.6-1.0) to indicate readiness for study involvement. To prevent rater drift, bi-weekly group coding sessions are conducted throughout the study period. The majority of sessions will be independently coded by two 
raters, to re-establish interrater reliabilities throughout the study duration. Rater ICCs and interventionist MITI 3.1 scores are examined throughout the study to determine if re-training of interventionists or raters is indicated. Retraining is indicated if rater ICCs fall below 0.6 or if interventionist ratings fall below competency thresholds outlined in the MITI 3.1. ${ }^{35}$

\section{Analyses}

Power Analyses-Power analyses were conducted to detect a significant difference in attrition between NOURISH ${ }^{+}$and NOURISH+MI (Aim 1) using PROC POWER in SAS v9.2. With a sample size of 60 individuals per group (the targeted recruitment number calculated below) and 0.64 as the reference proportion (those who completed the postintervention in the NOURISH pilot), we have $80 \%$ power ( $a=0.05$, two-sided) to detect a 0.21 difference in proportion of participants completing post-intervention between the groups. As we anticipate that NOURISH+MI will have greater retention, we should have power to detect a smaller difference in proportions. For the main study outcome for Aim 2, child BMI percentile, 45 individuals per condition are required to detect a mean difference of 1.5 for a two-sided test, with power $=80 \%, a=0.05$ and $\mathrm{SD} \approx 2.5$ (based on NOURISH pilot data) between the NOURISH+MI and NOURISH ${ }^{+}$groups. We anticipate $80 \%$ retention, so will recruit 60 participants for NOURISH+MI. A simpler two-group model was used to estimate sample size; this was done because sample size calculations in the context of a model with a between subjects factor, a blocking factor, a within subjects factor, and a covariate would require assumptions about the effects of each of these which are not estimable.

Planned Statistical Analyses-We will first explore the data using descriptive statistics and graphical techniques. In addition, we will compare groups on child and parent ethnicity and gender. If significant group differences are found, the appropriate variables will be entered as covariates in the models.

The study's first aim is to pilot the feasibility and efficacy of a brief MI intervention as an adjunct to the RCT of NOURISH ${ }^{+}$, and examine the impact of NOURISH+MI on attrition and adherence. To address this aim, chi-square analyses will examine differential attrition at post-test and follow up and t-tests will assess differences in attendance at treatment sessions between conditions (NOURISH ${ }^{+}$and NOURISH+MI). The second and third aims are to examine the efficacy of NOURISH+MI on child and parent BMI and BMI percentile (respectively), child and parent dietary intake (kcals from the NDS-R analysis), and child and parent physical activity. For child BMI percentile, parent BMI, dietary intake and physical activity for children and parents, we will fit a repeated measures, mixed model design using hierarchical linear models and/or general estimating equations to account for the nested structure of the data. This model will include one between-subjects factor (treatment with 3 levels, control, NOURISH ${ }^{+}$, NOURISH+MI), one within-subjects factor (time with 3 levels, pre-test, post-test, and 4-month follow-up), and their interaction. If more than one child per family participates, models will be applied using a spatial autocorrelation matrix. ${ }^{53}$ All analyses will be applied using a modified intent-to-treat approach. ${ }^{54} \mathrm{We}$ will assess the assumption of dropouts being at random by comparing dropouts and completers on demographic and baseline variables. We will fit the model blind to treatment and 
examine measures of model fit (AIC, BIC/SBC) to evaluate whether a simpler covariance structure is reasonable; if so, we will use that before breaking the blind and examining fixed effects.

Last, our fourth aim is exploratory and will identify putative mediators and moderators of treatment outcomes. To address this aim, we will evaluate the influence of putative moderators (e.g., ethnicity and age of children, measured at baseline) and mediators (e.g., parent BMI, readiness to change) on outcomes by conducting first order analyses. Second order analyses will add the significant moderators/mediators, and/or interactions between these variables and treatment group, to the models used for Aims 2 and 3. Although underpowered, results will inform future translational research based upon this proposed study and the R01 (NOURISH ${ }^{+}$) findings.

\section{Discussion}

Although it is recommended that health professionals incorporate MI into pediatric obesity treatments, more rigorous investigations are warranted, particularly among parents of overweight children. ${ }^{21,26,55,56}$ Strengths of NOURISH+MI include its design (RCT), target population (primarily African American families of low socioeconomic status), focus on parents, and emphasis on treatment integrity. Further, this study design is innovative as it uses a cost-effective method of building off a larger trial. Results of both trials will then inform subsequent applications in this scientific area.

The addition of two brief ( 1 telephone, 1 in-person) MI sessions does not significantly increase respondent burden, and will also allow us to investigate two modes of intervention delivery. The dose of this adjunctive treatment is small. In our previous work, $M I$ Values, ${ }^{30,57}$ a 2-session adjunctive MI intervention was associated with significantly improved dietary intake and improved adherence among obese, primarily African American adolescents enrolled in a multidisciplinary treatment program. Recently, MacDonnell et al. ${ }^{56}$ noted that two MI sessions might be effective as an adjunct to more intensive programs to elicit weight loss, based on their findings with African American, obese adolescents. Other studies have demonstrated that MI interventions with only one session were effective in improving outcomes. ${ }^{58,59}$ If effective, NOURISH+MI will contribute to the development of strategies for addressing major problems in pediatric obesity research: attrition, suboptimal outcomes, and low treatment engagement. ${ }^{10}$ Moreover, because NOURISH ${ }^{+}$targets parents alone, we will examine use of MI exclusively with parents of overweight children. In this way, this study responds to the significant question regarding with whom to intervene when implementing MI in pediatric obesity treatment (parent and/or child). ${ }^{26,60,61}$ Future research should continue to investigate the appropriate target of MI (parent[s], child, and/or both) within pediatric obesity treatments across ages and developmental stages.

We expect that MI will improve treatment engagement, as it is a patient centered and nonjudgmental approach which uses parents' own values and goals to increase readiness to change. ${ }^{62}$ This strategy contrasts with more confrontational, fear-based, or informationgiving approaches that are frequently used in healthcare, which tend to elicit greater resistance to change (e.g., warning about the dangers of not changing, highlighting increased 
disease risk, or providing information without tailoring it to the patient's needs and selfefficacy). ${ }^{22}$ MI builds parents self-efficacy for change, highlights their autonomy, and affirms their current strengths. In these ways, we anticipate that, after participating in MI, parents' readiness to engage in treatment and make behavioral changes will increase as their ambivalence will be reduced (or resolved).

Timing of MI was informed by previous studies demonstrating that adding MI early in the intervention process increases adherence to program goals and improves outcomes. ${ }^{21}$ Indeed, it is recommended that MI interventions be implemented as a "prelude to treatment" to diffuse ambivalence about treatment engagement. ${ }^{56}$ For example, Golberg et al. ${ }^{59}$ implemented a single MI session prior to randomization into a behavioral weight management program for obese adults; although they had no control group, retention was high (96\%), far exceeding typical results in weight management studies. We will be able to examine if effects are similar among parents of children with overweight.

To increase internal validity, recruitment, assessments, screening, and group intervention procedures for NOURISH+MI participants are identical to those in $\mathrm{NOURISH}^{+}$(using trained interventionists, adhering to the Operations Manual, and monitoring fidelity). Groups only differ in number of MI sessions: two for NOURISH+MI and none for NOURISH ${ }^{+}$. To minimize contamination, NOURISH+MI participants attend independent orientation sessions and groups from participants enrolled in $\mathrm{NOURISH}^{+}$. Attention differs between groups, such that the NOURISH+MI participants have more contact with interventionists ( 40 minutes). However, we will be able to examine if this brief additional contact using MI is a beneficial, cost and time effective approach to enhance treatment effects within a parent intervention for pediatric overweight. Further, although we are only conducting MI with the treatment arm of $\mathrm{NOURISH}^{+}$, we will be able to adapt this MI approach easily in future studies and apply it to both conditions (control or $\mathrm{NOURISH}^{+}$) at enrollment, similar to that described by Goldberg et al., ${ }^{59}$ to enhance retention and reduce differential attrition.

A limitation of this study involves the use of 24 hour diet records to assess dietary intake. However, NOURISH+ (and thus NOURISH+MI) investigators selected this method over more cost and labor-intensive, high cognitive burden approaches as a feasible, valid and cost-effective strategy for assessing dietary intake (which is a secondary study outcome). ${ }^{63-65}$ Lastly, while the main trial of NOURISH+ has an additional assessment at 10-month follow up, NOURISH+MI follow-up is limited to 4-months post-intervention due to the 2-year timeline and budget of the funding mechanism, prohibiting examination of sustained changes and/or relapse beyond this period. While longer-term data are needed, we decided to include two follow-up periods (post-testing and 4-month follow-up) due to their consistency with the larger NOURISH ${ }^{+}$trial and the feasibility within our funding source. We will then be able to use these pilot data, and data from the $\mathrm{NOURISH}^{+}$trial, to inform future translational, community-based work.

\section{Summary}

Obesity, physical inactivity, and dietary factors are major contributors to poor health.

Current recommendations suggest that health professionals integrate MI into pediatric obesity treatments, as an addition to other behavior change strategies; there is a clear need 
for more rigorous investigations in this area, particularly among African American families. ${ }^{21,26,55,56}$ Innovative strategies to improve treatment adherence and reduce attrition are critical to improve the efficacy of pediatric obesity interventions. When implemented as part of an obesity treatment, MI might represent a cost- and time effective strategy to enhance treatment engagement. This study will capitalize on the concurrent trial of $\mathrm{NOURISH}^{+}$and examine an adjunctive intervention within this larger RCT, comparing the MI arm with the two treatment arms from the larger trial (NOURISH ${ }^{+}$and control), representing a cost-effective research design. We hypothesize that this adjunctive MI intervention will enhance treatment effects through reduced attrition and better adherence with $\mathrm{NOURISH}^{+}$, a parent intervention culturally tailored for African American parents of overweight children. Results have the significant potential to enhance understanding of the application of MI to pediatric obesity treatment and to inform future translational studies.

\section{Acknowledgments}

The NOURISH+MI study is supported by a grant from the American Heart Association [13CRP1457008 to MK Bean]; NOURISH ${ }^{+}$is funded by the National Institutes of Health [R01HD066216-01A1 to SE Mazzeo]. These funding sources had no involvement with the conduct of the research or preparation of the manuscript. This trial is registered with ClinicalTrials.gov NCT01912989

\section{References}

1. Kiess W, Galler A, Reich A, et al. Clinical aspects of obesity in childhood and adolescence. Obes Rev. 2001; 2:29-36. [PubMed: 12119634]

2. Sorof J, Daniels S. Obesity hypertension in children: a problem of epidemic proportions. Hypertension. 2002; 40:441-7. [PubMed: 12364344]

3. Schwimmer JB, Burwinkle TM, Varni JW. Health-related quality of life of severely obese children and adolescents. Jama. 2003; 289:1813-9. [PubMed: 12684360]

4. Whitaker RC, Wright JA, Pepe MS, Seidel KD, Dietz WH. Predicting obesity in young adulthood from childhood and parental obesity. N Engl J Med. 1997; 337:869-73. [PubMed: 9302300]

5. McGovern L, Johnson JN, Paulo R, et al. Clinical review: treatment of pediatric obesity: a systematic review and meta-analysis of randomized trials. J Clin Endocrinol Metab. 2008; 93:46005. [PubMed: 18782881]

6. Oude Luttikhuis H, Baur L, Jansen H, et al. Interventions for treating obesity in children. Cochrane Database Syst Rev. 2009:CD001872. [PubMed: 19160202]

7. Stern M, Mazzeo SE, Gerke CK, Porter JS, Bean MK, Laver JH. Gender, ethnicity, psychosocial factors, and quality of life among severely overweight, treatment-seeking adolescents. J Pediatr Psychol. 2007; 32:90-4. [PubMed: 16818482]

8. Jeffery RW, Drewnowski A, Epstein LH, et al. Long-term maintenance of weight loss: current status. Health Psychol. 2000; 19:5-16. [PubMed: 10709944]

9. Karlson CW, Rapoff MA. Attrition in Randomized Controlled Trials for Pediatric Chronic Conditions. Journal of Pediatric Psychology. 2009; 34:782-93. [PubMed: 19064607]

10. Skelton JA, Beech BM. Attrition in paediatric weight management: a review of the literature and new directions. Obesity Reviews. 2010:e273-e81. [PubMed: 20880126]

11. U.S. Department of Health and Human Services NIH. Strategic Plan for NIH Obesity Research. 2011. In

12. Kitzmann KM, Dalton WT 3rd, Stanley CM, et al. Lifestyle interventions for youth who are overweight: a meta-analytic review. Health Psychol. 2010; 29:91-101. [PubMed: 20063940]

13. Golan M, Kaufman V, Shahar DR. Childhood obesity treatment: targeting parents exclusively v. parents and children. Br J Nutr. 2006; 95:1008-15. [PubMed: 16611394] 
14. Janicke DM, Sallinen BJ, Perri MG, Lutes LD, Silverstein JH, Brumback B. Comparison of program costs for parent-only and family-based interventions for pediatric obesity in medically underserved rural settings. J Rural Health. 2009; 25:326-30. [PubMed: 19566621]

15. Boutelle KN, Cafri G, Crow SJ. Parent-only treatment for childhood obesity: a randomized controlled trial. Obesity. 2011; 19:574-80. [PubMed: 20966907]

16. Koplan JP, Liverman CT, Kraak VI. Preventing childhood obesity: health in the balance: executive summary. J Am Diet Assoc. 2005; 105:131-8. [PubMed: 15635359]

17. Mazzeo SE, Gow RW, Stern M, Gerke CK. Developing an intervention for parents of overweight children. International Journal of Child and Adolescent Health. 2008; 1:355-63.

18. Mazzeo SE, Kelly NR, Stern M, et al. Nourishing our understanding of role modeling to improve support and health (NOURISH): Design and methods. Contemporary Clinical Trials. 2012; 33:515-22. [PubMed: 22273843]

19. Bean MK, Wilson DB, Thornton LM, Kelly N, Mazzeo SE. Dietary intake in a randomizedcontrolled pilot of NOURISH: A parent intervention for overweight children. Prev Med. 2012; 55:224-7. [PubMed: 22735041]

20. Miller, WR.; Rollnick, S. Motivational Interviewing: Preparing People for Change. Guilford Press; New York: 2002.

21. Hettema J, Steele J, Miller WR. Motivational Interviewing. Annual Review of Clinical Psychology. 2005; 1:91-111.

22. Emmons KM, Rollnick S. Motivational interviewing in health care settings. Opportunities and limitations. American Journal of Preventive Medicine. 2001; 20:68-74. [PubMed: 11137778]

23. Suarez M, Mullins S. Motivational interviewing and pediatric health behavior interventions. J Dev Behav Pediatr. 2008; 29:417-28. [PubMed: 18852613]

24. Martins RK, McNeil DW. Review of Motivational Interviewing in promoting health behaviors. Clinical Psychology Review. 2009; 29:283-93. [PubMed: 19328605]

25. Schwartz RP, Hamre R, Dietz WH, et al. Office-based motivational interviewing to prevent childhood obesity: a feasibility study. Arch Pediatr Adolesc Med. 2007; 161:495-501. [PubMed: 17485627]

26. Resnicow K, Davis R, Rollnick S. Motivational interviewing for pediatric obesity: Conceptual issues and evidence review. J Am Diet Assoc. 2006; 106:2024-33. [PubMed: 17126634]

27. VanWormer JJ, Boucher JL. Motivational interviewing and diet modification: a review of the evidence. Diabetes Educ. 2004; 30:404-16. [PubMed: 15208841]

28. Taveras EM, Gortmaker SL, Hohman KH, et al. Randomized Controlled Trial to Improve Primary Care to Prevent and Manage Childhood Obesity: The High Five for Kids Study. Archives of Pediatrics \& Adolescent Medicine. 2011 doi.10.1001/archpediatrics.2011.44.

29. Bean MK, Mazzeo SE, Stern M, Bowen D, Ingersoll K. A values-based Motivational Interviewing (MI) intervention for pediatric obesity: study design and methods for MI Values. Contemp Clin Trials. 2011; 32:667-74. [PubMed: 21554994]

30. Bean MK, Powell P, Quinoy A, Ingersoll K, Wickham Iii EP, Mazzeo SE. Motivational interviewing and adherence to pediatric obesity treatment: Results from the MI Values randomized controlled trial. Pediatric Obesity under revision.

31. Bandura, A. Social foundations of thought and action: a social cognitive theory. Englewood Cliffs, N.J.; Prentice-Hall: 1986.

32. Faith MS, Van Horn L, Appel LJ, et al. Evaluating Parents and Adult Caregivers as "Agents of Change" for Treating Obese Children: Evidence for Parent Behavior Change Strategies and Research Gaps. Circulation. 2012; 125:1186-207. [PubMed: 22271754]

33. Ammerman A, Leung MM, Cavallo D. Addressing disparities in the obesity epidemic. N C Med J. 2006; 67:301-4. [PubMed: 17066662]

34. Mazzeo, SE.; Kelly, NR.; Stern, M., et al. Eating Behaviors in press. Parent skills training to enhance weight loss in overweight children: Evaluation of NOURISH.

35. Moyers, TB.; Martin, JK.; Manuel, J.; Miller, WR.; Ernst, D. Revised Global Scales: Motivational Interviewing Treatment Integrity 3.1.1 (MITI 3.1.1). 2010.

Contemp Clin Trials. Author manuscript; available in PMC 2015 March 01. 
36. Bean MK, Mazzeo SE, Stern M, Bowen D, Ingersoll K. A Values-Based Motivational Interviewing (MI) Intervention for Pediatric Obesity: Study Design and Methods for MI Values. Contemporary Clinical Trials. 2011; 32:667-74. [PubMed: 21554994]

37. Berg-Smith SM, Stevens VJ, Brown KM, et al. A brief motivational intervention to improve dietary adherence in adolescents. The Dietary Intervention Study in Children (DISC) Research Group. Health Educ Res. 1999; 14:399-410. [PubMed: 10539230]

38. Miller, WR.; C'de Baca, J. Quantum Change: When Epiphanies and Sudden Insights Transform Ordinary Lives. Guilford Press; New York: 2001.

39. Rhee KE, De Lago CW, Arscott-Mills T, Mehta SD, Davis RK. Factors associated with parental readiness to make changes for overweight children. Pediatrics. 2005; 116:e94-101. [PubMed: 15995022]

40. Kristal AR, Glanz K, Curry SJ, Patterson RE. How can stages of change be best used in dietary interventions? Journal of the American Dietetic Association. 1999; 99:679-84. [PubMed: 10361529]

41. Birch LL, Fisher JO, Grimm-Thomas K, Markey CN, Sawyer R, Johnson SL. Confirmatory factor analysis of the Child Feeding Questionnaire: a measure of parental attitudes, beliefs and practices about child feeding and obesity proneness. Appetite. 2001; 36:201-10. [PubMed: 11358344]

42. Sallis JF, McKenzie TL, Alcaraz JE. Habitual physical activity and health-related physical fitness in fourth-grade children. Am J Dis Child. 1993; 147:890-6. [PubMed: 8352224]

43. Gross LD, Sallis JF, Buono MJ, Roby JJ, Nelson JA. Reliability of interviewers using the SevenDay Physical Activity Recall. Res Q Exerc Sport. 1990; 61:321-5. [PubMed: 2132889]

44. Feskanich D, Buzzard IM, Welch BT, et al. Comparison of a computerized and a manual method of food coding for nutrient intake studies. Journal of the American Dietetic Association. 1988; 88:1263-7. [PubMed: 3171019]

45. Feskanich D, Sielaff BH, Chong K, Buzzard IM. Computerized collection and analysis of dietary intake information. Comput Methods Programs Biomed. 1989; 30:47-57. [PubMed: 2582746]

46. Neumark-Sztainer D, Eisenberg ME, Fulkerson JA, Story M, Larson NI. Family meals and disordered eating in adolescents: longitudinal findings from project EAT. Archives of pediatrics \& adolescent medicine. 2008; 162:17-22. [PubMed: 18180407]

47. Centers for Disease Control and Prevention. [Accessed January 17, 2006Accessed January 17, 2006] National Center for Health Statistics. Clinical Growth Charts. 2000. Available at: http:// www.cdc.gov/growthcharts/at http://www.cdc.gov/growthcharts/

48. Samet, JM.; Alberg, AJ. Surrogate sources of dietary information. In: Willett, W., editor. Nutritional Epidemiology. Oxford University Press; New York: 1998. p. 157-73.

49. Varni JW, Seid M, Kurtin PS. PedsQL 4.0: reliability and validity of the Pediatric Quality of Life Inventory version 4.0 generic core scales in healthy and patient populations. Med Care. 2001; 39:800-12. [PubMed: 11468499]

50. Varni JW, Seid M, Rode CA. The PedsQL: measurement model for the pediatric quality of life inventory. Med Care. 1999; 37:126-39. [PubMed: 10024117]

51. Taylor SJ, Whincup PH, Hindmarsh PC, Lampe F, Odoki K, Cook DG. Performance of a new pubertal self-assessment questionnaire: a preliminary study. Paediatr Perinat Epidemiol. 2001; 15:88-94. [PubMed: 11237120]

52. Horvath AO, Greenberg LS. Development and Validation of the Working Alliance Inventory. Journal of Counseling Psychology. 1989; 36:223-33.

53. Cheng A, Kelly P. Application of a hierarchical model incorporating intrafamily correlation and cluster effects. Nursing Research. 2011; 60:208-12. [PubMed: 21317823]

54. Spilker, B. Guide to Clinical Trials. Raven Press, Ltd.; New York: 1991.

55. Barlow SE. Expert committee recommendations regarding the prevention, assessment, and treatment of child and adolescent overweight and obesity: summary report. Pediatrics. 2007; 120(Suppl 4):S164-92. [PubMed: 18055651]

56. MacDonnell K, Brogan K, Naar-King S, Ellis D, Marshall MD. A Pilot Study of Motivational Interviewing Targeting Weight-Related Behaviors in Overweight or Obese African American Adolescents. Journal of Adolescent Health. 2012; 50:201-3. [PubMed: 22265118] 
57. Powell, P.; Mazzeo, SE.; Stern, M.; Bean, MK. Society for Pediatric Psychology. San Antonio; TX: Motivational interviewing (MI) in a multidisciplinary pediatric obesity treatment: Preliminary findings from MI Values.

58. McCambridge J, Strang J. The efficacy of single session motivational interviewing in reducing drug consumption and perceptions of drug-related risk and harm among young people: results from a multi-site cluster randomized trial. Addiction. 2004; 99:39-52. [PubMed: 14678061]

59. Goldberg JH, Kiernan M. Innovative techniques to address retention in a behavioral weight-loss trial. Health Educ Res. 2005; 20:439-47. [PubMed: 15598664]

60. Erickson SJ, Gerstle M, Feldstein SW. Brief interventions and motivational interviewing with children, adolescents, and their parents in pediatric health care settings: a review. Arch Pediatr Adolesc Med. 2005; 159:1173-80. [PubMed: 16330743]

61. Daniels SR, Jacobson MS, McCrindle BW, Eckel RH, Sanner BM. American Heart Association Childhood Obesity Research Summit Report. Circulation. 2009; 119:e489-517. [PubMed: 19332458]

62. Rollnick, S.; Miller, WR.; Butler, CC. Motivational Interviewing in Healthcare: Helping Patients Change Behavior. The Guilford Press; New York: 2008.

63. Buzzard, M. 24-hour dietary recall and food record methods. In: Willett, W., editor. Nutritional Epidemiology. Oxford Press; New York: 1998. p. 50-73.

64. Baxter SD. Cognitive processes in children's dietary recalls: insight from methodological studies. Eur J Clin Nutr. 2009; 63(Suppl 1):S19-32. [PubMed: 19190640]

65. Lytle LA, Nichaman MZ, Obarzanek E, et al. The CATCH Collaborative Group. Validation of 24hour recalls assisted by food records in third-grade children. Journal of the American Dietetic Association. 1993; 93:1431-6. [PubMed: 8245378] 


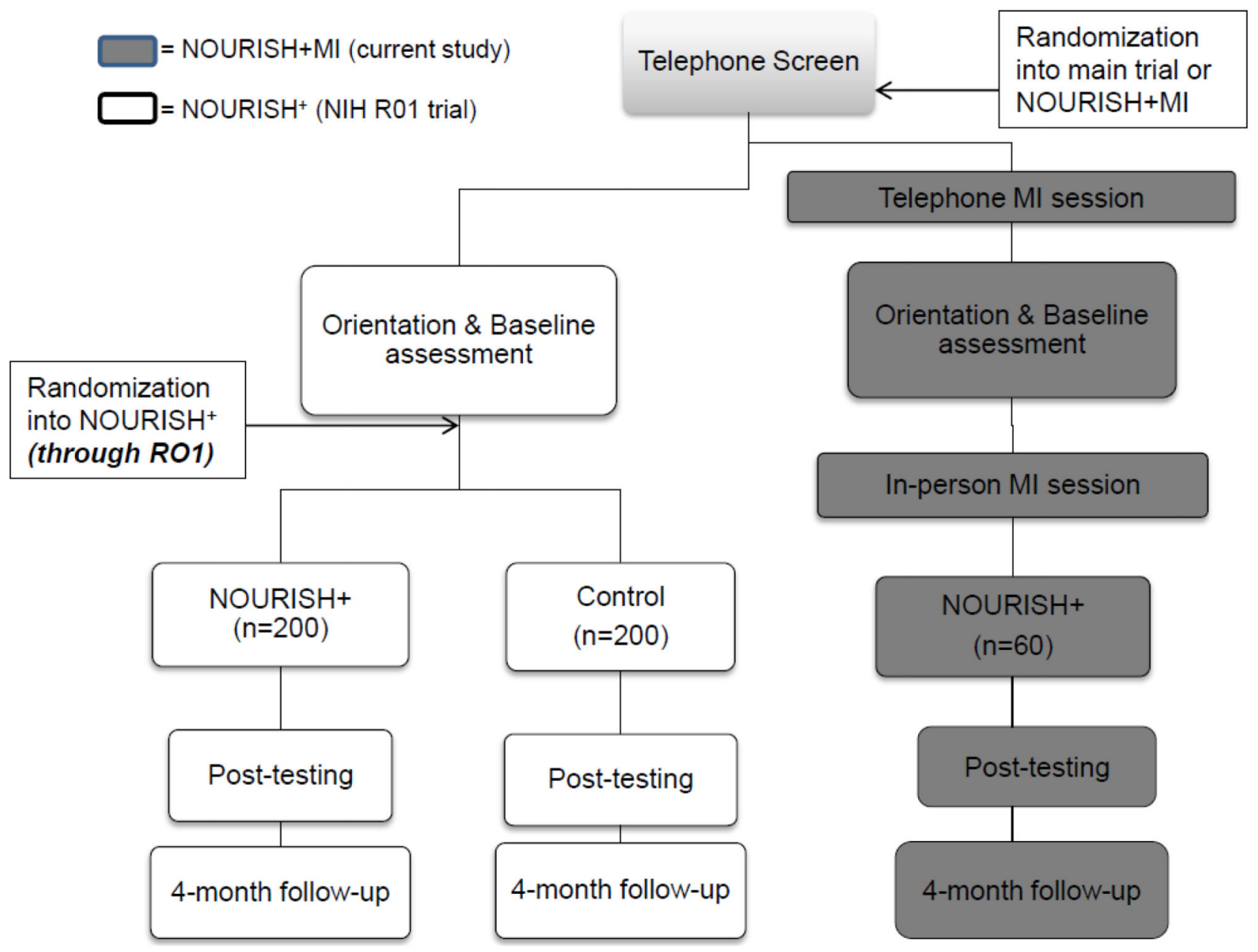

Figure 1. Flowchart of NOURISH+MI as an adjunctive treatment arm to $\mathrm{NOURISH}_{+} \mathrm{R01}$ 


\section{Identifying Personal Values}

Sometimes people find that identifying their personal values is helpful when making important life decisions and changes, such as taking steps toward a healthier family with the NOURISH ${ }^{+}$ Program. The following exercise may help you to clarify your values and how they relate to your family's current eating and exercise behaviors. Place a checkmark next to those values that are most important to you. There is also space provided to write in values that are not listed, if desired.

$\square$ Service
$\square$ Self-control
$\square$ Simplicity
$\square$ Order/control
$\square$ Responsibility
$\square$ Caring/compassionate
$\square$ Commitment
$\square$ Cooperation
$\square$ Love
$\square$ Moderation
$\square$ Honesty
$\square$ Humility
$\square$ Knowledge
$\square$ Generosity
$\square$ Health
$\square$ Helpfulness
$\square$ Dependability
$\square$ -

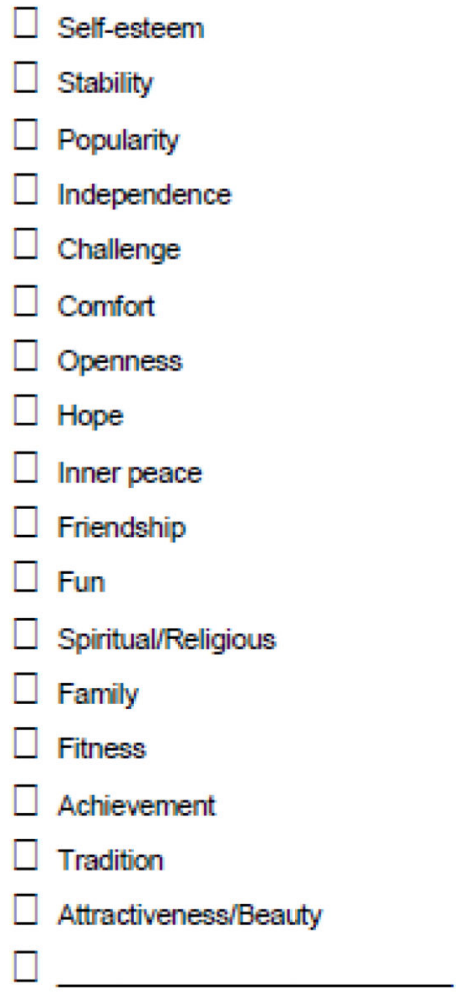

Now select your top three values from the list above and write them on the lines below.

1.

2.

3.

Figure 2. 


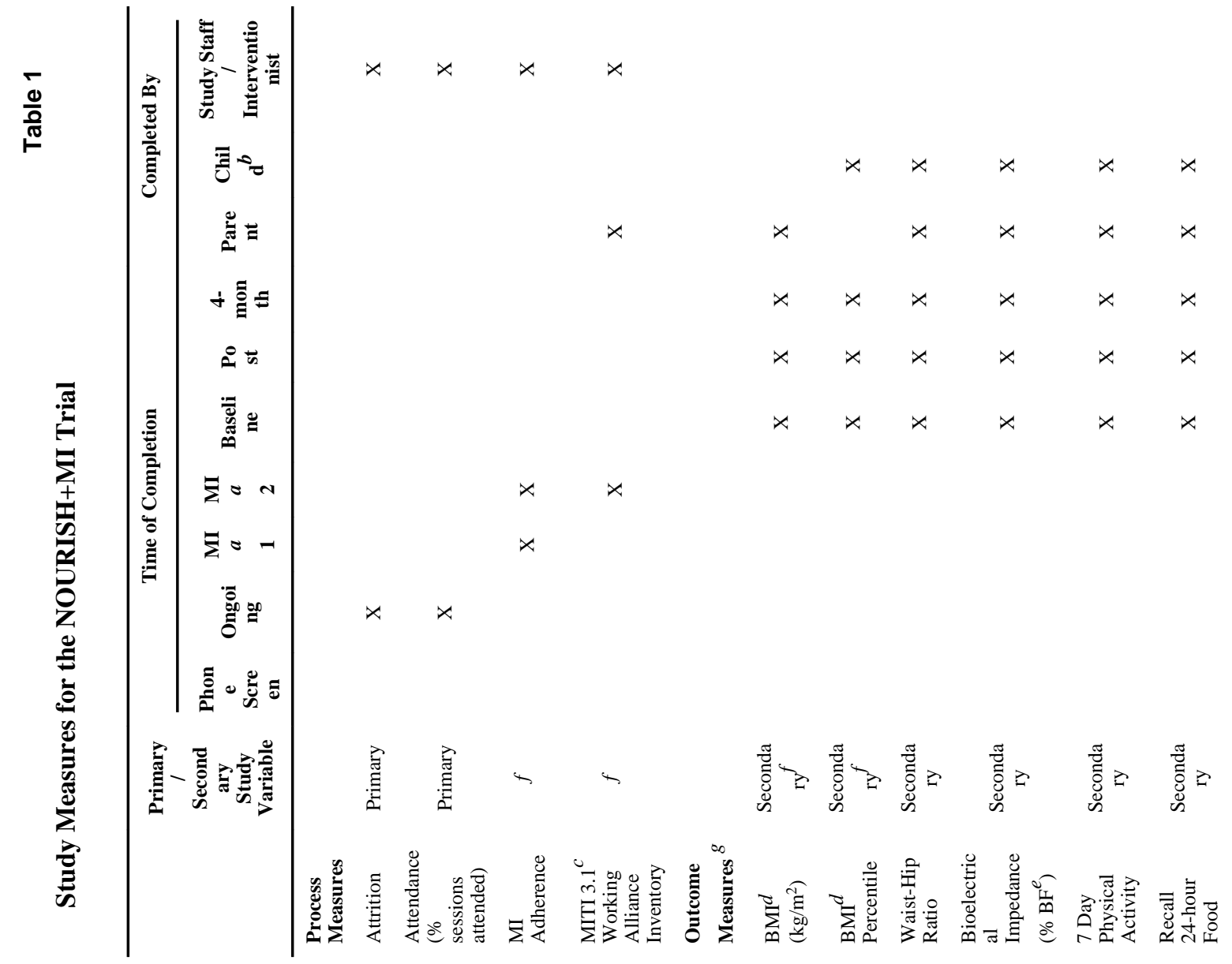

Contemp Clin Trials. Author manuscript; available in PMC 2015 March 01. 


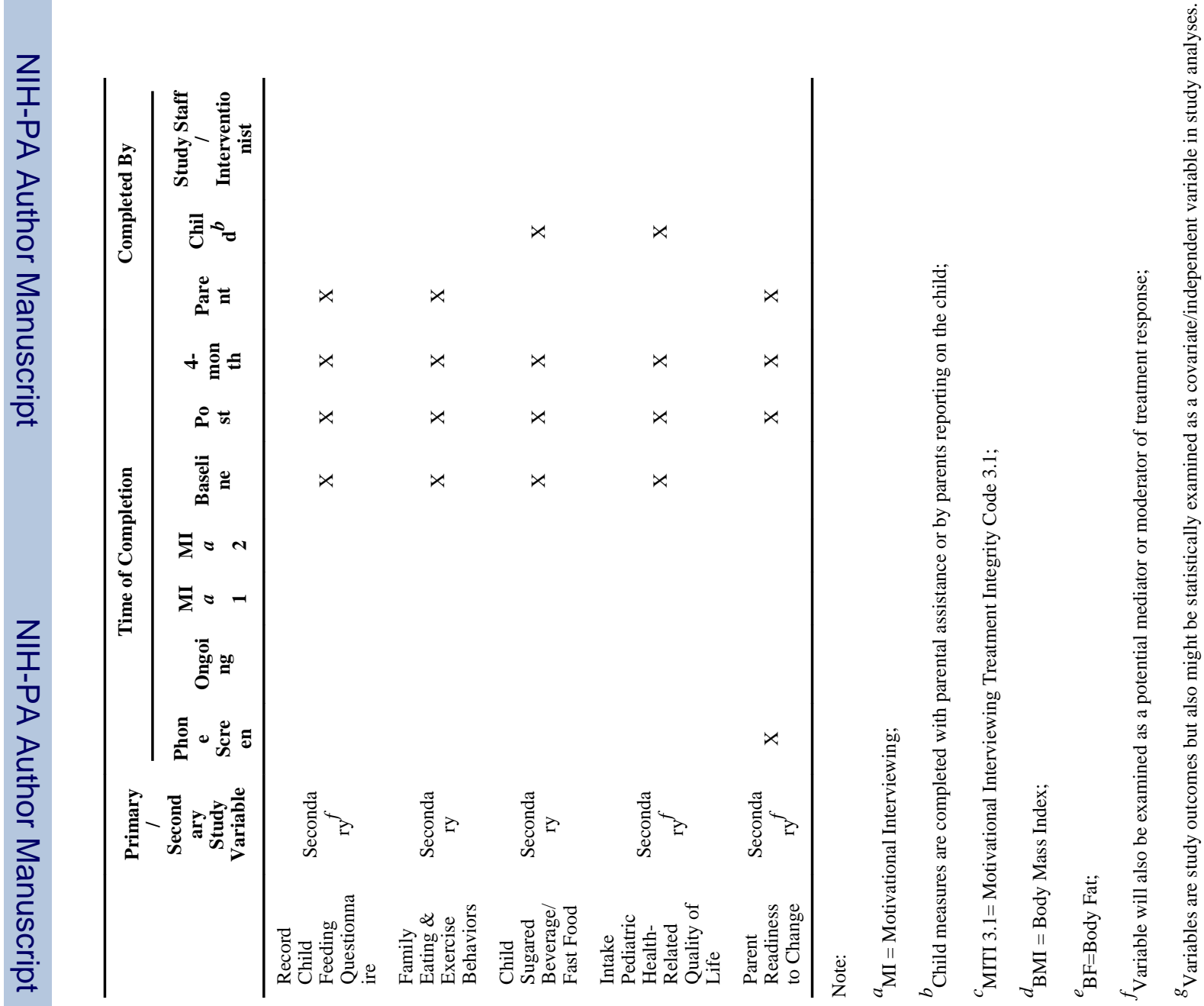


Table 2

Motivational Interviewing Proficiency at Study Onset Compared with Recommended Proficiencies Using the MITI 3.1 ${ }^{a}$

\begin{tabular}{|c|c|c|c|}
\hline \multirow[b]{2}{*}{ MITI Domain } & \multirow{2}{*}{$\begin{array}{c}\text { Mean Rating } b \\
M(S D)\end{array}$} & \multicolumn{2}{|c|}{ MITI 3.1 Recommended Proficiencies } \\
\hline & & Beginner & Competent \\
\hline Global Spirit ${ }^{c}$ & $4.8(0.27)$ & 3.5 & 4 \\
\hline Reflection:Question ${ }^{d}$ & $1.8(1.09)$ & 1.0 & 2.0 \\
\hline$\%$ Complex Reflections ${ }^{e}$ & $87.9(0.11)$ & $40 \%$ & $50 \%$ \\
\hline$\%$ Open Questions $^{f}$ & $69.2(0.17)$ & $50 \%$ & $70 \%$ \\
\hline$\%$ MI Adherent ${ }^{g}$ & $100(0.00)$ & $90 \%$ & $100 \%$ \\
\hline
\end{tabular}

${ }^{a}$ MITI 3.1 = Motivational Interviewing Treatment Integrity Code, Version 3.1

${ }^{b}$ Means represent ratings from 4 interventionists across 21 encounters

${ }^{c}$ Global Spirit $=($ Evocation + Collaboration + Autonomy $) / 3$

$d_{\text {Ratio }}=$ Total Reflections/Total Questions

$e_{\%}$ Complex Reflections $=($ Complex Reflections $/$ Total Reflections $) \times 100$

$f_{\%}$ Open Questions $=($ Open Questions/Total Questions $) \times 100$

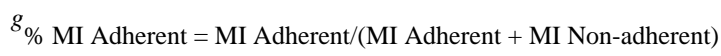

